\title{
Sobre la oportunidad de la rehabilitación psicosocial tras un primer brote: un caso clínico
}

RESUMEN: El inicio de las intervenciones rehabilitadoras en las fases tempranas de la psicosis, o tras un primer brote, ha generado un importante número de investigaciones así como el desarrollo de programas y la organización de servicios específicos.

PALABRAS CLAVE: Esquizofrenia, primeros brotes, período crítico, intervención temprana.
SUMMARY: The psychosocial rehabilitation in early phases of psychosis or after a first episode of psychosis has generated a lot of investigation and the implement of programs and organization of specific services.

KEY WORDS: Schizophrenia, first episodes, critic period, early intervention.

\section{Introducción}

En los últimos años, varios autores destacan en su investigación los beneficios de comenzar el proceso de rehabilitación psicosocial tras el primer brote psicótico. Argumentan que el comienzo temprano de la intervención ha demostrado ser más eficaz que la intervención en estados más avanzados de la enfermedad. Esta hipótesis se fundamenta teóricamente en el concepto de «periodo crítico».

El «periodo crítico» puede definirse como «aquel período de la esquizofrenia en el que existe una máxima sensibilidad a las influencias biológicas y sociales» (Birchwood, y otros, 1998). Esta fase, que abarcaría los dos o tres primeros años posteriores al primer episodio psicótico, se caracterizaría tanto por una mayor progresión del deterioro, como por una mayor sensibilidad a las intervenciones terapéuticas (1). Se considera que éste es el momento de mayor plasticidad biológica, psicológica y psicosocial, por lo que parece que las acciones rehabilitadoras que se lleven a cabo en este periodo determinarán en gran medida la evolución de la enfermedad y el pronóstico a largo plazo del paciente. Como se trata de una fase en la que la influencia de los factores externos es mayor, la intervención obtendrá mejores resultados y requerirá menos esfuerzos que en los casos en los que la rehabilitación se lleva a cabo en etapas más tardías. También parece demostrado que el tratamiento en esquizofrenias de mayor duración se relaciona con resultados más pobres y que los retrasos en la instauración de la intervención pueden suponer una evolución más pobre.

Se ha llegado a afirmar que este periodo en el que se producen cambios biológicos, psicosociales y cognitivos decisivos para el curso de la enfermedad puede llegar incluso a predecir con un importante margen de seguridad el curso a largo plazo de una persona con esquizofrenia (1). 
Además, el curso inicial de la psicosis es inestable y proclive a las recaídas, con un $80 \%$ de pacientes que recaen en los primeros 5 años de enfermedad. En este momento, son también habituales la exacerbación de los síntomas, el consumo de drogas, las respuestas desadaptativas de evitación o negación de la enfermedad, el mantenimiento de una sintomatología positiva residual y el incremento del riesgo de suicidio (1). Por ello, se han desarrollado algunas opciones terapéuticas que pretenden cubrir estas carencias, adaptándose a las necesidades específicas de este período. Las opciones terapéuticas existentes más importantes y que están generando mayor cantidad de estudios de investigación son (2):

1. La Terapia de Orientación Cognitiva para la Psicosis Temprana «COPE» (Jackson, y otros, 1999; Henry, y otros, 2002), que se desarrolló con el objetivo de ayudar al paciente en la recuperación inicial de su primer episodio psicótico y de evitar o aliviar la frecuente morbilidad secundaria asociada a éste. Pretende ayudar a preservar un sentido del yo (identidad), promover una sensación de dominio sobre la experiencia psicótica y preservar o incrementar la autoeficacia ante el inicio de la enfermedad. Tiene un formato individual, se inicia al final de la fase aguda del episodio psicótico, consta de entre 20 y 30 sesiones y utiliza una combinación de psicoeducación y de técnicas cognitivas (3).

2. El Tratamiento Sistemático de la Psicosis Persistente «STOPP» (HermanDoig, y otros, 2003; Jackson, y otros, 1999), que va destinada a las personas que presentan una recuperación prolongada del primer episodio de psicosis. Es de orientación cognitiva, con un formato individual y tiene como objetivos principales reducir la frecuencia e intensidad de los síntomas positivos, reducir el malestar y la interferencia con el funcionamiento normal producidos por la sintomatología psicótica residual, reducir el trastorno emocional y promover en el individuo una comprensión de la psicosis que permita su participación activa en la regulación del riesgo de recaída y de la discapacidad social (4).

Por otro lado, existen programas de intervención temprana que están formados habitualmente por equipos interdisciplinares, que proporcionan una amplia serie de servicios integrados que suelen incluir educación pública, evaluaciones amplias y rápidas, manejo clínico de casos e intervenciones grupales. Las intervenciones específicas incluyen farmacoterapia, psicoeducación, manejo de estrés, prevención de recaídas, solución de problemas, consejo y apoyo, y rehabilitación social y laboral (2).

El centro decano de la intervención temprana, que ha servido de modelo para múltiples iniciativas internacionales que han surgido posteriormente es El Centro de Prevención e Intervención en Psicosis Temprana (EPPIC), desarrollado para tratar las necesidades de pacientes en las primeras fases de un episodio psicótico. Se trata de un servicio multicomponente cuyo objetivo es la reducción del nivel de 
morbilidad primaria y secundaria en los primeros episodios psicóticos, tratando de identificar lo más tempranamente posible a los pacientes tras el inicio de la psicosis (5).

Otro de los servicios específicos para jóvenes con un primer episodio psicótico que también ha desempeñado un papel fundamental para el desarrollo actual de los servicios de intervención temprana es El Servicio de Intervención Temprana de Birmingham (EIS), que está formado por equipos interdisciplinares de urgencia psiquiátrica y tratamiento domiciliario 24 horas al día, servicios de enlace y atención primaria, seguimiento activo y servicios de recuperación y rehabilitación (6).

Las intervenciones integrales procedentes de los servicios de intervención temprana han demostrado que mejoran el curso inicial de la enfermedad, desapareciendo prácticamente el tiempo de espera sin recibir tratamiento de los pacientes en sus primeros episodios psicóticos (Larsen, y otros, 2001) (7), a la vez que mejoran el estado clínico, el funcionamiento social, y reducen las rehospitalizaciones y la duración de las estancias hospitalarias (Mc Gorry, y otros, 1996; Addington, y otros, 2003; Petersen, y otros, 2005) $(5 ; 8 ; 9)$. Aún así, estos primeros resultados de los estudios de investigación hay que contemplarlos con cautela, debido a las múltiples carencias metodológicas de los mismos, y constituyendo la propia organización de los servicios de intervención temprana aún un tema abierto (2).

En cuanto a lo que las intervenciones psicosociales se refieren, McDonald, y otros (1998) encuentran en pacientes con primeros brotes que aquellos con mayores sentimientos de autoeficacia, mayor percepción de apoyo social y estrategias de afrontamiento del estrés más flexibles, afrontan mejor las situaciones estresantes habituales y por lo tanto presentan menor riesgo de recaer. Todos estos factores parecen potenciarse a través de una intervención psicosocial (10). Por último, Albiston, y otros (1998) han estudiado la eficacia de un programa de intervención grupal en primeros brotes, centrado en intervenciones psicosociales que faciliten la adaptación del paciente a su trastorno y su integración social, obteniendo tras seis meses de seguimiento mejores niveles de ajuste social y de síntomas negativos. Estos y otros resultados parecen demostrar claramente la conveniencia y la rentabilidad de una intervención psicosocial temprana (11).

En conclusión, las investigaciones parecen indicar que la intervención psicosocial: puede mejorar con más probabilidad el curso deteriorante de las psicosis si se aplica desde los momentos iniciales de la enfermedad. Puede prevenir el deterioro psicológico y social. Produce mejores resultados con la misma cantidad de intervención.

Si se mantiene durante todo el periodo crítico, al «relajar» dicha intervención no se retrocede hasta el nivel inicial. Se puede conseguir una mejor adaptación y recuperación de la psicosis en la fase postayuda. 
Existen algunos factores que parecen favorecer la recuperación. Así, la existencia de redes adecuadas de apoyo social, un entorno seguro y estructurado y una situación de vida estable son algunos de ellos. También parece importante poder alcanzar una buena comprensión de lo que ha ocurrido, tener expectativas realistas y esperanza en el futuro o disponer de una meta u objetivo en la vida (trabajo, actividades de ocio, etc.).

La probabilidad de que estos aspectos se encuentren conservados o menos deteriorados es mayor cuando el periodo de enfermedad es corto. El ajuste psicológico y los roles sociales y laborales están en ese momento más cercanos a la normalidad a pesar incluso de la ruptura o el impacto que supone haber padecido un primer episodio psicótico. Además, las primeras crisis se producen frecuentemente en la adolescencia o juventud, periodo en el que existe una alta sensibilidad a la alteración de las distintas áreas de funcionamiento que se están empezando a desarrollar. Esto supone que el retraso en el tratamiento en esta etapa vital puede tener consecuencias muy negativas: interrupción en los estudios o en el trabajo, pérdida de apoyos sociales y de la autoestima, mayor angustia en la familia del paciente; en definitiva una interferencia grave en el desarrollo psicosocial. Todo ello supone una recuperación más lenta y menos completa (y por lo tanto un peor pronóstico), así como un aumento en el coste del tratamiento (12).

Así, los objetivos de la rehabilitación tras un primer brote serían los siguientes: a) disminuir la discapacidad a largo plazo; b) minimizar el impacto de la psicosis en los roles que el usuario venía ejerciendo o, en su caso, recuperación de dichos roles; c) mejorar la actitud ante el tratamiento; d) disminuir el estrés y el impacto que supone la experiencia del primer episodio psicótico y los primeros contactos con los servicios psiquiátricos; e) remisión de los síntomas positivos persistentes y de los síntomas negativos; f) promover la sensación de control sobre las consecuencias de la enfermedad a través de la mejora de los recursos para afrontar situaciones estresantes; g) proteger la autoestima y separar el «yo» o el sentido de identidad del proceso psicótico; h) disminuir la percepción de amenaza sobre las perspectivas futuras tanto sociales como educativo/laborales; i) aumentar el conocimiento de todo lo relacionado con la enfermedad y prevenir recaídas; j) evitar la ruptura de relaciones sociales y, en su caso, facilitar el establecimiento de otras nuevas; k) apoyar a la familia.

En definitiva, se trataría de promover la mejor adaptación posible y la recuperación funcional y psicosocial.

Uno de los aspectos más importantes en el trabajo tras un primer brote es la «psicoeducación». Por un lado, el desconocimiento de lo que está ocurriendo supone un alto estrés para la persona que ha padecido la crisis. Por otro, este conocimiento tiene una gran importancia para la prevención de recaídas. Reconocer las 
primeras señales de un episodio psicótico y de los factores de riesgo personales que aumentan la vulnerabilidad puede atajar una nueva crisis o atenuar sus efectos. Además de reducir el riesgo de una recaída precoz y de facilitar la adaptación ante la crisis que supone padecer un trastorno psicótico y requerir tratamiento por primera vez, la psicoeducación persigue facilitar la aceptación del tratamiento farmacológico y psicosocial, y conseguir que la persona acepte un apoyo continuo $(13 ; 14)$.

Otra cuestión fundamental, como ya se ha mencionado, sería evitar la pérdida de las habilidades sociales, académicas o profesionales y «preservar roles y competencias» que se tenían antes de la crisis (y que están más conservadas que en casos de mayor evolución). Esto puede favorecerse tratando de modificar la pérdida de confianza en uno mismo y la sensación de desorganización que suele seguir al episodio, evitando la inactividad, la pérdida de capacidades, la dependencia y la separación de los grupos sociales previos. El objetivo final sería que el paciente se reintegre en su medio y no rompa con sus nexos de apoyo $(13 ; 14)$.

En un principio, estas metas pueden promoverse tanto de forma individual como por medio de la inclusión de la persona en «programas de grupo» destinados a mejorar diversas áreas de funcionamiento. Aunque en el trabajo de rehabilitación existen grupos con objetivos muy diversos, el trabajo grupal tendría en estas primeras etapas algunos beneficios generales: ofrecen la oportunidad para evitar la inactividad y fomentar la independencia de la familia; muchos de ellos implican el desarrollo o puesta en práctica de habilidades sociales y mantienen la sensación de pertenencia a un grupo social. Esto supone adquirir una sensación de control sobre el entorno en grupos menos estresantes o exigentes y en los que la sensación de apoyo y de aceptación es menor. Con todo ello, se consigue además que la sensación de estigma disminuya. Por otro lado, el contacto con personas en distintas fases del proceso de recuperación puede alentar una visión más positiva de futuro y permite ver cuánto se puede mejorar. Finalmente, los grupos pueden servir para aumentar la motivación para fijar y tratar de conseguir objetivos realistas y alcanzables $(13 ; 14)$.

Otro campo de trabajo importante sería la «rehabilitación laboral o académica», puesto que las oportunidades en este periodo son mayores que en casos de mayor evolución $(13 ; 14)$.

Finalmente, el «trabajo con las familias» es fundamental, no sólo porque su participación es necesaria para el progreso del paciente, sino por la necesidad que ellos mismos tienen de apoyo y orientación $(13 ; 14)$. A la vez, se debe considerar que las necesidades familiares iniciales son diferentes a las de la familia cuando ya lleva años conviviendo con la enfermedad, puesto que en estos momentos todavía carecen de experiencia alguna con la misma, el diagnóstico inicial suele ser 
ambiguo y la recuperación inicial de un primer episodio suele ser buena en un porcentaje elevado de pacientes, lo cual desaconseja intervenciones pensadas para cuidados crónicos más continuados (Addington y Burnett, 2004) (15).

Por otro lado, los conceptos tradicionales de emoción expresada pueden ser en estos casos menos relevantes que en las familias de pacientes con periodos más largos de enfermedad y cobran mayor importancia el manejo de la situación de crisis y alto estrés. Es probable encontrar en estos familiares sentimientos de negación, pena, estigma, fracaso, vergüenza o cólera.

Así, los aspectos más específicos del trabajo con estas familias serían: maximizar el funcionamiento adaptativo de la familia. Minimizar la disrupción de la vida familiar causada por el episodio psicótico. Minimizar el riesgo de desarrollar sufrimiento, estrés y carga a largo plazo. Ayudar a la familia a entender el impacto de la psicosis en todos y cada uno de sus miembros así como su influencia en el curso de la misma. Promover una sensación de control sobre la experiencia (Addington; Collins; McCleery; Addington, 2005) (16).

Otros objetivos importantes serían: apoyarles a nivel emocional, ayudarles a establecer expectativas y objetivos realistas, entrenarles en formas adecuadas de interactuar con el paciente y enseñarles a crear un entorno estructurado y de bajo estrés, tratando de reducir al mínimo cualquier cambio en la rutina. En definitiva, dotarles de recursos para afrontar y controlar los problemas que caracterizan la situación por la que están pasando.

Finalmente, el hecho de facilitar el contacto con otras familias tras un primer brote puede evitar que se produzca aislamiento y fomenta el apoyo emocional $(2 ; 17)$.

Expondremos a continuación el trabajo realizado en el Centro de Rehabilitación Psicosocial de Alcalá de Henares (en adelante CRPS) con un usuario que había sufrido un primer brote psicótico, y que ilustra los principios expuestos en las líneas anteriores.

\section{Caso clínico}

Datos biográfico-clínicos

Acude al CRPS desde el mes de junio de 2002. Tiene 17 años en el momento de la evaluación. El parto y desarrollo evolutivo fueron normales. Con un año el usuario sufre tres episodios de convulsiones febriles producidas por gastroenteritis que se resuelven sin complicaciones.

Vive con los padres en casa de la abuela materna en Madrid. A los dos años y medio acude a la escuela infantil durante tres meses y a los cuatro años comien- 
za el colegio. Es la abuela la que se encarga de su cuidado, ya que los padres trabajaban. Durante la etapa escolar tiene buen rendimiento académico, pero se relacionaba poco con los compañeros. Se relacionaba fundamentalmente con un vecino que iba al mismo colegio. Parece que este niño insultaba y agredía con frecuencia al usuario, que incluso refiere haber sufrido abusos sexuales bajo amenaza, aunque ni el colegio ni la familia lo sabía. A los 13 años empieza el Instituto y comienza a salir con un grupo de chicos con los que juega a hacer «espiritismo»; cuando todos los demás abandonan el juego porque les da miedo, deja de relacionarse con ellos. Con esta edad refiere que comienza a oír ruidos extraños por la noche que posteriormente se transforman en voces que le dan órdenes. No se lo cuenta a nadie. Paulatinamente, disminuye considerablemente su rendimiento académico. Pasó dos años en esta situación, hasta que un año antes de su llegada al CRPS le cuenta a su madre que le están espiando y observando. Poco antes de que lo expresara le notaban más angustiado, tímido y preocupado. Comienza así su tratamiento psiquiátrico, y presenta en ese momento algunos episodios de agresividad, sobre todo contra objetos y en algunas ocasiones a personas. Deja de acudir al Instituto y consigue una profesora que le ayuda con los estudios desde casa. También acude a diario a un Hospital de Día hasta ser derivado al CRPS.

Desde el CSM se realiza un diagnóstico de esquizofrenia paranoide. Es ingresado en dos ocasiones en la Unidad de Adolescentes del Hospital General Universitario Gregorio Marañón. Desde el alta del segundo ingreso se instaura tratamiento farmacológico con Leponex. Los síntomas principales que padece hasta el segundo ingreso son trastornos de la identificación y alucinaciones auditivas y visuales complejas muy frecuentes. Posteriormente, y en el momento de la evaluación, la sintomatología consiste en embotamiento afectivo, pensamientos recurrentes sobre su enfermedad y sobre la posibilidad de hacer daño a alguien o a sí mismo, ánimo depresivo, alucinaciones visuales y auditivas (que disminuyeron en frecuencia e intensidad), aislamiento social y falta de interés por las actividades.

Tiene el título de Graduado en Educación Secundaria. Repite 4..$^{\circ}$ curso y lo acaba en el año 2002 gracias al apoyo recibido por la organización no gubernamental «Save the Children». Estudió durante cinco años ofimática y creación de páginas web y durante cuatro años inglés, ambos en una academia privada. Nunca ha trabajado.

El usuario vive en casa con sus padres, su hermana de 10 años y su abuela materna que recientemente se ha trasladado a vivir con ellos. En el momento de la evaluación, la red social del usuario se limitaba a una sola persona, un amigo que le presentaron y con el que salía los fines de semana. Presentó muchas dificultades para relacionarse con otros desde la infancia. Sus dificultades incluían tanto aspectos no verbales (le costaba mantener la mirada y tenía un tono de voz muy 
bajo) como problemas para iniciar y mantener conversaciones con personas desconocidas. Esto ha provocado que nunca haya tenido una red estable. Además, muchas de las situaciones que desencadenaban los síntomas positivos eran precisamente situaciones sociales que el paciente no sabía resolver o no identificaba correctamente (por ejemplo, siempre que veía un grupo de chicos de su edad, pensaba que se estaban riendo de él).

En cuanto a las relaciones familiares, no se detectaron signos de criticismo u hostilidad en la familia. Existía una cierta sobreprotección de la madre, quizá debido a la edad y problemática del usuario. Éste dice confiar mucho en sus padres, sobre todo en su madre, a la que le cuenta todo. Cuando tiene alucinaciones en la calle su forma de resolverlo es correr a casa a contárselo a su madre. Parece que hay buenas relaciones de apoyo y confidencialidad por parte de todos, incluida la abuela. La encargada de poner límites a Rubén es su madre. Su padre se mantiene más periférico, sobre todo en lo que tiene que ver con la enfermedad. La familia realiza muchas actividades de ocio juntos (parque de atracciones, salidas al campo, etc.).

Conoce la ciudad, se mueve en transporte público con total autonomía. Es autónomo en el acceso a recursos. Sus hábitos de aseo, sueño y alimentación son adecuados y normalizados. Nunca ha consumido tóxicos. Identifica señales de ansiedad en contextos relacionados con dos situaciones: estar con otras personas y el Instituto. Dentro de éstas refiere: si va en autobús sin ser acompañado por su madre. Cuando se cruza por la calle con otras personas, aunque sea un sitio conocido. Cuando va a discotecas que ponen música «bakalao». Cualquier acercamiento al Instituto. La situación que define como más ansiógena es pasar al lado de chicos de su edad que se están riendo, sobre todo cuando está en el Instituto.

No se detectan conductas alteradas o problemáticas. Había presentado algún episodio de agresividad verbal y física en el contexto familiar, pero en el momento de la evaluación no había vuelto a presentar ninguno desde hacía tiempo. Sus capacidades básicas están totalmente conservadas, aunque sí presenta muchos efectos secundarios de la medicación que le producían ciertas dificultades cognitivas.

El «Plan Individualizado de Rehabilitación» que se estableció tras la evaluación fue el siguiente:

\section{Prevención de posibles crisis.}

1.1. Que aumente sus conocimientos sobre la enfermedad y las variables implicadas.

1.2. Que aprendan a detectar pródromos tanto él como su familia.

1.3. Que aprenda a controlar los episodios heteroagresivos.

1.4. Que tome la medicación de forma autónoma. 
DEBATES E INFORMES

2. Que adquiera mayor nivel de habilidades que le permitan mantener y aumentar la red social.

2.1. Disminuir el nivel de ansiedad social con grupos de iguales.

2.2. Que adquiera habilidades para mantener conversaciones.

2.3. Que tenga respuestas más asertivas.

\section{Integración Comunitaria.}

3.1. Que pueda ir solo por la ciudad.

3.2. Que coja transportes urbanos (autobuses, etc.).

3.3. Que conozca recursos de ocio.

\section{Integración formativo-laboral.}

4.1. Que retome los estudios formales en el Instituto en horario nocturno.

En cuanto a la «INTERVENCIÓN», para facilitar su organización, la hemos dividido por áreas, a pesar de la dificultad que ello conlleva:

\section{Prevención de crisis/psicoeducación}

Aunque el paciente conocía el diagnóstico y se había informado en Internet sobre la enfermedad, cuando llegó al centro presentaba muchas dudas. Por ello se le informó de las características de la esquizofrenia, los síntomas, su curso y las distintas fases de la enfermedad. Se le explicó también el modelo de vulnerabilidad-estrés, tratando de que identificara los factores de riesgo que podrían provocar una recaída, así como los signos tempranos de una nueva crisis.

No pudimos identificar o reconocer cuáles habían sido los pródromos del brote psicótico que sufrió y que requirió que ingresara en dos ocasiones por persistir la sintomatología de manera importante, probablemente porque cuando fue diagnosticado ya llevaba varios años oyendo voces. Parece que el comienzo de la enfermedad fue insidioso.

Los síntomas positivos fueron muy frecuentes e intensos desde que se produjo el diagnóstico, aunque remitieron en cierta medida cuando comenzó el tratamiento con Olanzapina. En este caso era especialmente importante que pudiera alcanzar un mayor control sobre esos síntomas, que consistían principalmente en episodios de alucinaciones auditivas y visuales muy complejas que llegaban a ser escenas que le provocaban un alto nivel de angustia y temor. Esos episodios eran lo que el paciente llamaba «crisis». 
En un primer momento se intervino sobre estos episodios mediante registros para identificar estímulos antecedentes de tales episodios, que consistían habitualmente en situaciones que le provocaban ansiedad, como ir en autobús o pasar al lado de un grupo de chicos de su edad, o en situaciones en los que se encontraba aislado, como cuando pasaba mucho tiempo en su habitación. Estos episodios iban seguidos habitualmente de conductas de evitación consistentes en salir corriendo en busca de su madre, incluso cuando ésta se encontraba fuera de casa, o de solicitar a su psiquiatra aumentos del tratamiento antipsicótico o incluso el ingreso.

El control de estos síntomas se llevó a cabo inicialmente de tres maneras:

1. Identificación de estas experiencias como un síntoma de su enfermedad y entrenamiento para que pudiera reconocer que no tenían carácter real.

2. Reestructuración cognitiva: se le pedía que buscara en el entorno evidencias que pudieran confirmar que lo que estaba viendo o escuchando era una alucinación.

3. Resolución de problemas: una vez analizadas las evidencias que apoyaban la explicación de las alucinaciones como un síntoma, se le pedía que generara otras alternativas de respuesta a la situación diferentes a la del escape. Una de esas alternativas era permanecer en la situación y exponerse a la misma en vez de correr y buscar a su madre, hasta conseguir que la ansiedad fuese disminuyendo.

De esta manera y junto con el tratamiento farmacológico se consiguió que disminuyeran la frecuencia y la intensidad de estos episodios, aunque seguían presentes de manera muy compleja ante situaciones que le generaban ansiedad y que resultaban ser de índole interpersonal.

En una fase más avanzada de la intervención, se trabajó sobre la identificación, por parte del usuario, de situaciones cotidianas que le generaban malestar, sobre las emociones y pensamientos implicados y la relación entre dichas situaciones y la aparición de los síntomas, con el fin de que pudiera comprender el funcionamiento de los mismos y adoptar estrategias que evitaran su aparición. Se pudo poner de manifiesto que las situaciones que desencadenaban los síntomas estaban muy relacionadas con aspectos de índole interpersonal, en los que estaban implicados sentimientos y pensamientos de rechazo o miedo a no ser aceptado, desconfianza y minusvaloración.

En el momento actual, ha presentado de forma puntual algún episodio de ansiedad, sin componente alucinatorio o delirante, ante situaciones de estrés, como el examen de selectividad. El usuario plantea abiertamente situaciones que le generan malestar, el tipo de malestar que experimenta, y como afronta las mismas, bien manteniéndose en la situación aceptando que es inevitable experimen- 
tar ansiedad en determinados momentos o bien poniendo en marcha estrategias de resolución de problemas o de habilidades asertivas.

Por otro lado, es realmente llamativa la disminución del temor del usuario a la reducción gradual de la medicación antipsicótica que le ha ido planteando su psiquiatra, lo que tiene que ver directamente con la confianza que ha adquirido en que los síntomas (a los que ahora denomina «esos pensamientos raros que me vienen cuando tengo ansiedad») se han ido reduciendo de forma proporcional al aumento de la confianza en sí mismo y en sus capacidades para afrontar cualquier tipo de problema, que evidentemente proviene de la exposición y afrontamiento de las situaciones que anteriormente le generaban un gran malestar.

Precisamente, los sentimientos de autoeficacia están estrechamente relacionados con el trabajo que se ha llevado a cabo en la siguiente área de intervención.

\section{Habilidades sociales}

En este caso mejorar las habilidades sociales era fundamental y eran consideradas una variable protectora, pues desde un principio se pensó que los síntomas podrían disminuir cuando las habilidades sociales fueran mejoradas.

La intervención perseguía conseguir una mejor percepción y afrontamiento de situaciones sociales, considerándose el objetivo final crear una red social más amplia y estable.

Para ello, se ha trabajado mediante su inclusión en un grupo de habilidades sociales, con un formato estándar, en el que se entrenaron las habilidades básicas de comunicación no verbal y verbal para iniciar, mantener y cerrar conversaciones.

Mediante esta intervención mejoraron notablemente sus capacidades para iniciar y mantener conversaciones en los distintos contextos de relación con un menor nivel de ansiedad, aunque seguía evitando en gran medida el contacto con personas de entornos normalizados y de edades parecidas a la suya. Por ello, desde tutorías y desde un grupo de habilidades sociales, esta vez con un formato muy específico y adaptado a los problemas de índole interpersonal que presentaban sus miembros, se empezó a trabajar el objetivo de aumentar su competencia social, incluyendo habilidades asertivas más complejas, en grupos de iguales en entornos normalizados (en su caso del Instituto donde cursaba el bachillerato). El trabajo que se realizó desde el grupo, que estaba muy adaptado a las necesidades individuales de cada uno de sus miembros, se complementó con la intervención desde tutorías, en el que se apoyaba lo trabajado en el grupo y se trataban cuestiones más personales, relacionadas con el miedo que tenía a relacionarse con grupos de iguales por su experiencia anterior tanto el colegio como el instituto en los que sufrió 
abusos por parte de compañeros de clase y vecinos del barrio. El objetivo consistía en que pudiera elegir o mostrarse en disposición de tener otro tipo de experiencias con iguales.

De esta manera, en el momento actual, el usuario mantiene un buen nivel de competencia social, incluyendo capacidades asertivas tales como resolver problemas interpersonales, pedir cambios, hacer críticas, decir que no, y expresar emociones tanto positivas como negativas en sus relaciones. Expresa abiertamente que ha recuperado la confianza en sí mismo y en los demás, que su miedo a relacionarse provenía de las experiencias de abuso que sufrió en el pasado, y que esas experiencias ya no condicionan su disposición a relacionarse con otros chicos y chicas de su edad.

Asimismo, ha aumentado su red social, tanto a través del Centro de Rehabilitación Psicosocial, donde mantiene amistad desde hace varios años con varios chicos, como fuera del mismo, en el Instituto y en la Universidad, mostrándose abierto a cualquier tipo de relación social. En conexión con este punto, resulta llamativo el cambio en su aspecto personal, que en un principio era bastante llamativo (ropas muy oscuras, caminar encorvado y arrastrando los pies, cabeza agachada) para pasar posteriormente a usar prendas de vestir de otros colores, caminar más erguido, no agachar la cabeza y disminuir en gran medida su rigidez corporal.

\section{Control de la ansiedad}

El paciente presentaba ansiedad, como ya se ha señalado, ante varias situaciones, siendo la ansiedad máxima en aquellas que implicaban estar con otras personas de su edad o estar en el instituto. El control de la ansiedad se trabajó en grupo mediante el entrenamiento en respiración abdominal y en autoinstrucciones positivas. Él refiere que el aprendizaje de estas técnicas le ha resultado útil para disminuir el nivel de ansiedad en los períodos de exámenes y para afrontar mejor las situaciones sociales. El afrontamiento de la ansiedad, unido a la mejora de sus habilidades sociales y la puesta en práctica de las mismas en numerosas y variadas situaciones reales estarían estrechamente interrelacionadas y serían las responsables de los logros mencionados anteriormente.

\section{Integración comunitaria}

La integración comunitaria del paciente era muy pobre. Conocía pocos sitios y recursos de su ciudad y había dejado de ir al instituto, aunque una profesora par- 
ticular le daba clases en casa para que pudiera aprobar el curso. El trabajo en esta área se diseñó planificando un acercamiento progresivo a situaciones académicas y grupales. El objetivo final sería su reincorporación al Instituto.

El primer paso fue incluirle en grupos del centro en los que los contenidos estaban bastante alejados de lo académico y que suponían un estrés bajo por encontrarse entre compañeros con dificultades similares a las suyas.

Posteriormente se incorporó a otros grupos fuera del centro, en los que los contenidos estaban aún alejados de las materias académicas, lo cual seguía suponiendo un avance en el acercamiento gradual hacia el objetivo final de integración en la enseñanza académica reglada. El siguiente paso consistió en retomar las clases con una profesora particular, para, finalmente, incorporarse al Instituto en horario de tarde. Una vez incorporado al Instituto, el usuario ha ido logrando avances en su integración a lo largo de los tres años que ha estado en él cursando el bachillerato. Al principio se ausentaba mucho de clase, evitaba los tiempos de descanso por el miedo a relacionarse con los compañeros, se salía de algunos exámenes, no acudía a otros, etc., todo lo cual se ha ido modificando hasta llegar a conseguir una integración totalmente normalizada, tanto a nivel académico como social, en el último curso de bachillerato. Estos logros le llevaron a plantearse más metas personales y a tomar la decisión de iniciar una carrera universitaria, en concreto, la licenciatura de biología. A este respecto, el usuario se encuentra satisfecho de haber conseguido acceder a estudiar una carrera que a él le gustaba mucho, se ve capaz de asumir la responsabilidad que ello implica y se encuentra totalmente integrado en el ambiente universitario. Para ello, y a iniciativa propia, realizó unos cursos previos al inicio formal de las clases, lo cual le facilitó dicha integración, tanto en lo académico como en lo social. Tiene un grupo de amigos con los que habitualmente comparte sus tiempos de descanso, además de otras actividades que se proponen hacer fuera de la universidad.

Por otro lado, sigue manteniendo relación con el grupo de amigos que conoció a través de un grupo de ocio en el Centro, y con los que queda de forma habitual para salir y realizar distintas y variadas actividades de ocio, incluido algún viaje, tanto dentro como fuera de Alcalá. Todo ello sin ninguna intervención por parte del centro.

\section{Intervención familiar}

Los padres presentaban una gran necesidad de información sobre lo que le ocurría a su hijo. Esto se trabajó inicialmente a nivel individual y posteriormente incluyéndolos en un grupo de familias, al que sólo acudió su madre. 
Otro de los objetivos consistía en que pudieran mejorar el manejo de situaciones problemáticas que se podían producir en casa. Finalmente, también se pretendía que la familia promoviera la independencia del usuario. En el momento actual, la madre tiene un conocimiento adecuado de la enfermedad de su hijo. Además, ha disminuido el nivel de estrés en su casa y no parecen existir dificultades para manejar cualquier situación problemática derivada de la enfermedad. Por otro lado, el padre se ha mantenido al margen de la intervención, a pesar de la necesidad que siempre manifestaron el usuario y su madre con respecto a que también se interesara por conocer la problemática de su hijo. Por ello, al no ser posible trabajar con el padre, y siendo éste un punto importante para el usuario, se han ido trabajando con él las situaciones problemáticas que han ido surgiendo derivadas del desconocimiento del padre de la enfermedad. En definitiva, en la actualidad, el funcionamiento del usuario en su casa no difiere del de cualquier otro chico de su edad, y de la relación con sus padres podría decirse exactamente lo mismo.

\section{Consideraciones finales sobre el caso}

Muy al contrario de lo que suele ser habitual, el usuario aceptó desde un primer momento el diagnóstico de esquizofrenia, posiblemente porque por fin encontraba una explicación válida a todos los fenómenos sensoperceptivos que había estado experimentando desde hacía tiempo, y que le habían resultado muy angustiosos y atemorizantes. Por ello, desde un primer momento se mostró muy receptivo a toda la información sobre la enfermedad y las variables implicadas. El conocimiento de los síntomas, por otro lado, facilitó que dejara de otorgarle un carácter real a las alucinaciones tan complejas que sufría y facilitó que adoptara estrategias de afrontamiento más activas y distintas a la evitación o a la búsqueda de protección materna.

Asimismo, esto se reflejaba, también de forma poco habitual, en un temor importante a que su psiquiatra le redujera las dosis de medicación neuroléptica, temor que posteriormente, a medida que iba logrando sus objetivos y por tanto contaba con otros recursos de afrontamiento de situaciones problemáticas de distinta índole, se fue reduciendo, hasta llegar a desaparecer dicho temor e incluso plantearse que quizás otros fármacos de acción más ansiolítica le pudieran resultar efectivos para afrontar situaciones de alto estrés.

Posteriormente, la necesidad de explicar su malestar exclusivamente mediante la enfermedad se fue complementando, primeramente, y reduciendo, después, con una explicación más normalizada del efecto que tenían las situaciones que le 
generaban estrés. Esto facilitó la adopción de roles normalizados en áreas importantes de su vida (familia, amigos, estudios, etc.) y que no basara la construcción de su identidad sobre la enfermedad mental, si no sobre aspectos y capacidades más saludables de su persona.

En relación con lo anterior, y una vez dotado de las habilidades necesarias, comenzó a relacionarse primeramente con personas con enfermedad mental y de mayor edad que él, encontrándose muy cómodo, y arriesgándose después, de forma paulatina, a abrirse a otro tipo de relaciones sociales más igualitarias y normalizadas, hasta llegar a conseguir un nivel de integración social similar al de cualquier otro chico de su edad y «desmontándose» la creencia del peligro que podía suponer relacionarse con los demás.

Todo este proceso ha ido acompañado del establecimiento de metas personales cada vez más exigentes, llegando de nuevo a niveles totalmente normalizados, e incluso llegando a tomar decisiones sobre su futuro formativo con las que los profesionales nos solemos mostrar bastante conservadores por el riesgo que suponen al conllevar una importante carga de estrés.

Para terminar, nos gustaría señalar la importancia de tener en cuenta las necesidades, opiniones y decisiones sobre su propia vida que expresan los usuarios, a veces en contra de nuestros pronósticos, especialmente cuando la rehabilitación se inicia, como en este caso, en un período vital tan desafiante y complejo como es la adolescencia o inicio de la juventud.

\section{Conclusiones y discusión}

Tradicionalmente, la derivación al CRPS de un paciente diagnosticado de esquizofrenia se realizaba después de un largo camino en el que la persona ya había sido atendida en diversos dispositivos de la red de salud mental, que ofrecían una asistencia psiquiátrica, pero que no daban cabida a intervenciones más intensivas y específicas, sobre de todo de índole psicosocial, más ajustadas a estos primeros momentos de la enfermedad. Lo anterior favorecía la aparición de un mayor deterioro e impedía que el paciente se beneficiara de la intervención psicosocial en estas primeras fases.

Consideramos que una vez aparecida la enfermedad, el CRPS puede convertirse en el lugar idóneo donde realizar dichas intervenciones psicosociales tempranas, ajustándose, por un lado, a las necesidades y peculiaridades de una edad normalmente muy joven en la que todavía no se han llegado a establecer o se están empezando a perfilar metas vitales y roles importantes, y por otro, a las características individuales del usuario objeto de intervención. 
Los objetivos generales de la rehabilitación psicosocial (favorecer la recuperación, apoyar la integración social, fomentar la normalización y la autonomía, etc.) son compatibles con las necesidades que presentan las personas que acaban de recibir un diagnóstico de psicosis. Además, tal y como planteamos en la introducción de este artículo, los dos o tres primeros años de evolución de la enfermedad son especialmente sensibles a este tipo de intervenciones.

Así, según Vallina (2003) (17), habiéndose obtenido datos que favorecen la viabilidad de los procedimientos de la intervención temprana en entornos asistenciales públicos de la red sanitaria, estaríamos ante una nueva visión de la rehabilitación, donde ya no se trata de esperar estados residuales de la enfermedad para intervenir, sino de implantar tales procedimientos desde los momentos iniciales de la patología y romper de ese modo la falsa dicotomía denunciada por Shepherd (1998) (18) entre tratamiento y rehabilitación. Se trataría, en definitiva, de promover una óptica rehabilitadora a lo largo de todo el curso de la enfermedad, situando en sus primeras fases el momento del inicio de un proceso de rehabilitación que deberá acompañar a la persona a lo largo de su trayectoria vital intensificando o atenuando su intensidad en función del propio devenir individual y de los distintos avances científicos que están por llegar en relación con el tema que nos ocupa (17).

Este caso refleja la rentabilidad de la intervención psicosocial temprana desde un dispositivo de tercer nivel, como es un CRPS, para la recuperación de una persona recientemente afectada de psicosis.

\section{BIBLIOGRAFÍA}

(1) Birchwood, M.; TodD, P.; JACKsON, C., «Early Intervention in Psychosis. The Critical Period Hypothesis», British Journal of Psychiatry, 1998 (supl.172), pp. 53-59.

(2) VALLINA, O., y otros, «Estado actual de la detección e intervención temprana en psicosis», Apuntes de Psicología, 2006, 24, 1-3, pp. 185-221.

(3) Henry, L. P., y otros, «Cognitively Oriented Psychotherapy for First-Episode Psychosis (COPE): a Practitioner's Manual», Manual 4 in a Series of Early Psychosis Manuals, 2002, Melbourne, EPPIC.

(4) Herrmann-Doig, T.; Maude, D.; Edwards, J., Systematic Treatment of Persistent Psychosis (STOPP): A Psychological Approach to Facilitating Recovery in Young People with First Episode Psychosis, Londres, Martin Dunitz, 2003.

(5) McGorry, P. D., y otros, «EPPIC: An Evolving System of Early Detection and Optimal Management», Schizophrenia Bulletin, 1996, 22 (2), pp. 305-326.

(6) BIRCHWOOD, M., «Intervención temprana en psicosis: investigación, práctica clínica y reforma de los servicios en el Reino Unido» en Vázquez Barquero, J. L.; Crespo, V.; Herrán, A. (eds.), Las fases tempranas de la psicosis, Barcelona, Masson, 2005, pp. 145-159.

(7) LARSEN, T. K., y otros, «Shortened Duration of Untreated First Episode of Psychosis: Changes in Patient Characteristics at Treatment», American Journal of Psychiatry, 2001, 158, pp. 1.917-1.919. 
(8) Addington, J.; Leriger, E.; Addington, D., «Symptom Outcome 1 Year after Admision to an Early Psychosis Program», Canadian Journal of Psychiatry, 2003, 48, pp. 204-207.

(9) Petersen, L., y otros, «Improving 1-Year Outcome in First-Episode Psychosis. OPUS Trial», British Journal of Psychiatry, 2005, 187, supl. 48, pp. 98-103.

(10) MacDonald, E. M.; Pica, S.; McDonald, S., y otros, «Stress and Coping in Early Psychosis: Role of Symptoms Self-efficacy, and Social Support in Coping with Stress», British Journal of Psychiatry, 1998, 172, supl. 33, pp. 122-127.

(11) Albiston, D. J.; Francey, S. M.; Harrigan, S. M., «Group Programmes for Recovery from Early Psychosis», British Journal of Psychiatry, 1998, 172, supl. 33, pp. 117-121.

(12) LAHERA, G., y otros, «Rehabilitación psicosocial tras el primer brote esquizofrénico. La gran oportunidad», Revista de la Asociación Madrileña de Rehabilitación Psicosocial, 2004, 10, 18 19, pp. 30-36.

(13) Libermann, R. P., Rehabilitación integral del enfermo mental crónico, Barcelona, Martínez Roca, 1993.

(14) RodRíGUEZ, A. (coord.), Rehabilitación psicosocial de personas con trastornos mentales crónicos, Madrid, Pirámide, 1997.

(15) Addington, J.; Burnett, P., «Working with Families in the Early Stages of Psychosis», en Gleeson, J.; McGorry, P. D. (eds.), Psychological Interventions in Early Psychosis. A Treatment Handbook, Chichester, UK, Wiley, 2004, pp. 99-116.

(16) Addington, J., y otros, «The Role of Family Work in Early Psychosis, Schizophrenia Research, 2005, 79, pp. 77-83.

(17) Vallina, O., «Los orígenes de la cronicidad: intervenciones iniciales en psicosis», Revista de la Asociación Madrileña de Rehabilitación Psicosocial, 2003, 9, 15-16, edición especial de las IV Jornadas de la AMRP, «Los márgenes de la Rehabilitación», pp. 15-27.

(18) ShePHerd, G., «Developments in Psychosocial Rehabilitation for Early Psicosis», Internacional Clinical Psychopharmacology, 1998, 13, supl. 1, pp. 53-57.

* Isabel Baldor Tubet, Psicóloga Clínica; M. ${ }^{a}$ Carmen Jerez Álvarez, Psicóloga Clínica. Centro de Rehabilitación Psicosocial de Alcalá de Henares (Área 3 - Madrid). Gestión Técnica: Grupo Exter, S.A; Margarita Rullas Trincado, Psicóloga Clínica. Directora Centro de Rehabilitación Psicosocial de San Fernando de Henares (Área 2 - Madrid); Juan González Cases, Psicólogo Clínico. Director Centro de Rehabilitación Psicosocial «Alcalá de Henares» (Área 3 - Madrid). Gestión Técnica: Grupo Exter, S.A. «Plan de Atención Social a personas con enfermedad mental grave y crónica» de la Consejería de Familia y Asuntos Sociales de la Comunidad de Madrid.

Correspondencia: Isabel Baldor Tubet. Centro de Rehabilitación Psicosocial «Alcalá de Henares». C/ Pablo Coronel, 18. 28802 Alcalá de Henares. Telfs.: 918823884 / 918823984 / 619128686. E-mail: ibaldor@telefonica.net

** Fecha de recepción: 24-III-2008 (aceptado el 14-IV-2008). 